\title{
Using $\beta$ hCG, prolactin and IGFBP I single or in Pairs to diagnose PROM
}

\author{
Original \\ Mohammad Alaa Mohy El-Din El-Ghannam ${ }^{1}$, Hassan Tawfik Khairy', Mohammad \\ El Mandooh Mohammad ${ }^{1}$, Mohammed Saeed El Din El Safty ${ }^{1}$, Amira Mohammad \\ Zaki Abozeid ${ }^{2}$
}

${ }^{l}$ Department of Obstetrics and Gynecology, Faculty of Medicine, Ain-Shams University, Cairo

${ }^{2}$ El-Galaa Teaching Hospital, Egypt

\begin{abstract}
Background: When diagnosing premature rupture membranes is query, choosing the appropriate management needs additional confirmatory test. Based on their metabolomics, some biochemical markers have been proposed as diagnostic aids. A perfect test could be composed of combining more than one marker. Combining markers is not arbitrary as some combinations could inversely impact the diagnostic accuracy of the test.

Aim: Improving sensitivity and specificity of diagnosis of preterm premature rupture of membranes by combining quantitative insulin like growth factor binding protein 1 (IGFBP-1) with quantitative $\beta \mathrm{HCG}$ and/or quantitative prolactin in cervicovaginal fluid.

Materials and Methods: It is a cross sectional study. IGFBP-1, prolactin and $\beta$-HCG concentrations in vaginal fluid were examined in 180 patients with sure membrane state and the diagnostic accuracy of each marker and each combination was determined. The study was conducted between January 2018 and September 2018 in Ain-Shams University Maternity Hospital after the approval of the institutional ethics committee. Informed consent has been obtained from the patients before participation.

Results: The sensitivity, specificity, PPV, and NPV of Prolactin with cutoff point $11 \mu \mathrm{IU} / \mathrm{ml}$ are 84.4, 73.2, 73.8, and 79.5 , respectively, gives accuracy percentage $75.4 \%$. The sensitivity, specificity, PPV, and NPV of ILGFBP-1 with cutoff point $102 \mu \mathrm{IU} / \mathrm{ml}$ are $88.3,93.5,100$, and 69.4 respectively gives accuracy percentage $81.5 \%$. The sensitivity, specificity, PPV, and NPV of B-HCG with cutoff point $104 \mathrm{mIU} / \mathrm{mL}$ are 85.1, 83.9, 96.2, and 72.8 respectively gives accuracy percentage $79.9 \%$. The sensitivity, specificity, PPV, and NPV of the combination of IGFBP-1 and $\beta$-HCG are 78.4, 100, 100 and 64 respectively gives accuracy percentage 89.7. The sensitivity, specificity, PPV, and NPV of the combination of IGFBP-1 and prolactin are 78.4, 100, 100 and 68.7, respectively, gives accuracy percentage 84.2. The sensitivity, specificity, PPV, and NPV of the combination of $\beta$-HCG and prolactin are 79.8, 100, 100 and 63.6 respectively gives accuracy percentage 77.2.

Conclusion:Combining IGFBP-1 and $\beta-\mathrm{HCG}$ is the best diagnostic combination to detect amniotic fluid presence in vaginal fluid. Combined IGFBP-1 and prolactin offered very little improvement compared to IGFBP-1 alone. Adding prolactin to $\beta$-HCG decreased accuracy than each of the other two markers alone.
\end{abstract}

Key Words: $\beta$-HCG, insulin like growth factor binding protein 1 (IGFBP-1), premature rupture of membranes (PROM), prolactin

Received:03 January 2020, Accepted: 09 February 2020

Corresponding Author: Amira Mohammad Zaki Abozeid, El-Galaa Teaching Hospital, Egypt, Tel.: 01069491030, E-mail: abozeidamira7@gmail.com

ISSN: 2090-7265, November 2020, Vol.10, No. 4

\section{INTRODUCTION}

Metabolomics opens up new avenues for biomarker discovery in different branches of medicine, including perinatology ${ }^{[1]}$.

The preterm newborn is susceptible to numerous serious medical complications both early and later in life $\mathrm{e}^{[2]}$.
The maternal decidua and adjacent fetal membranes undergo anatomic and biochemical changes that ultimately result in a spontaneous rupture of the membranes at term. Premature activation of this mechanism due to PPROM leads to up to preterm deliveries ${ }^{[3]}$.

The dilemma about the rupture of membranes is attributed to the broad range of management choices it owns in dealing with ${ }^{[4]}$. 
Failure to identify women with PROM associated with failure to implement standard measures and infectious morbidities $^{[5]}$.

Delivering easy and accurate measure to diagnose PROM to primary care centers would help evading such hazardous morbidities to both mother and fetus by aiding decision of timely intervention and proper management, and would also help avoiding unnecessary cost and pitfalls of hospitalization, investigation and intervention when not needed ${ }^{[6]}$.

The primary problem in the diagnosis of PROM is to distinguish small amounts of amniotic fluid from other body fluids which may be present in the vagina. A number of non-invasive methods, based on; cytologic, biochemical or biophysical differences between amniotic fluid and other body fluids, have been proposed and used ${ }^{[7]}$.

The lack of a non-invasive "gold standard" test for the diagnosis of membrane rupture has mandated the search for alternative biochemical markers ${ }^{[8]}$.

Noninvasive cervicovaginal markers such as fFN, alpha fetoprotein, prolactin, human chorionic gonadotropin (hCG), placental $\alpha$-microglobulin 1 (PAMG-1), and insulin-like growth factor-binding protein 1 (IGFBP-1) have been studied for their ability to confirm or exclude membran Receiver-operator e rupture ${ }^{[4]}$.

Human chorionic gonadotropin (hCG) is secreted by the placental synctiotrophoblast cell layer ${ }^{[9]}$.

IGFBP1 is highly expressed during the stages between zygote and embryo phases, and nearly abolished in the normal adult organism ${ }^{[10]}$.

Prolactin is also synthesized in the decidualized stroma of endometrial tissue ${ }^{[1]}$.

When multiple tests are involved, the performance of the diagnosis may be improved by combining several tests as a new composite diagnostic test, since different tests may be sensitive to different aspects of case ${ }^{[12]}$.

Strategies for combining information from multiple diagnostic predictors are needed, since a combination may provide a better tool for diagnosis or screening applications than any single marker on its own ${ }^{[13]}$.

The choice of component tests and the rule for combining them strongly influences the accuracy of composite reference standards. Ideally, the combination of test results and the corresponding final diagnosis should be specified before the study to prevent data driven decisions. However, if there is uncertainty about the best composite reference standard, a sensitivity analysis could be planned to see how sensitive the results are to the particular choice of tests or combination rule ${ }^{[14]}$.

In the current thesis, we tested possible combinations of some biomarkers in order to enhance their accuracy. Those markers have been already investigated as diagnostic tools for equivocal cases of rupture membranes. In spite of being significantly diagnostic, none of them has been proven ideal yet.

\section{AIM OF THE WORK}

Improving sensitivity and specificity of diagnosis of preterm premature rupture of membranes by combining quantitative insulin like growth factor binding protein 1 (IGFBP-1) with quantitative $\beta \mathrm{HCG}$ and/or quantitative prolactin in cervico-vaginal fluid.

\section{PATIENTS AND METHODS}

Study type: It is a cross sectional study.

Study population : A total number of 180 patients with known membranes state between 28 to 36 weeks gestation of single viable fetus have been included in the study and have been categorized into two groups ; group I (the PROM group) inclued 90 patients with confirmed ruptured membranes and group II (control group) inclucded 90 patients with negative history of any leakage per vagina.

The study included 90 patients between 28 to 36 weeks gestation of single viable fetus with history of a leakage of clear water-like fluid from the vagina, which have been confirmed by visualization of leakage by sterile speculum in lithotomy position. Amniotic fluid index has been measured and documented by ultrasound, and compared to previous US if available.

Moreover, 90 patients between 28 to 36 weeks gestation of single viable fetus, who have been attending for their antenatal visits, with no history of any leakage of fluid from the vagina were recruited as control. They have been investigated by sterile speculum examination and amniotic fluid index by ultrasound have been included in the study as control group to confirm the cutoff point that was concluded by the researches that confirmed the usefulness of each marker (Figure 1).

Patients with any criterion that might alter the composition of leaked liquor like blood in vaginal secretion from any source, use of vaginal drugs or intercourse in the prior night, meconium in amniotic fluid, presence of fetal anomalies, intrauterine fetal death, diagnosed chorioamnionitis based on clinical factors 
such as ; maternal fever $>38^{\circ} \mathrm{C}$, uterine tenderness and maternal or fetal tachycardia or multiple pregnancies had been excluded.

Patients with risk factors that mandates expedition termination or cesarean section like fetal distress, placenta previa, medical complications that mandates termination of pregnancy or onset of active phase of labor in the form of regular, painful contraction and cervical dilatation $\geq 4 \mathrm{~cm}$ had been also excluded.

Sample size calculations : Considering the given accuracy in ${ }^{[7]}$ who investigated 88 patients divided into two groups for IGFBP-1, ${ }^{[15]}$ who tested prolactin in vaginal fluid of 114 women and ${ }^{[8]}$ tested qualitative human chorionic gonadotropin (hCG) on a total of 92 pregnant women and by using EPI-info 6 based on $95 \%$ confidence interval, power $80 \%$ and $P$ value $<0.05$ the sample size was calculated to be 90 patients with confirmed rupture membranes. A control group consists of 90 patients with intact membranes is taken to establish cut off values.

Ethics statement : The study was conducted between January 2018 and September 2018 in Ain-Shams University Maternity Hospital after the approval of the institutional ethics committee. Informed consent has been obtained from the patients before participation includes information about risks and benefits out of the research.

Study methods : All patients were subjected to determining gestational age based on the first day of the last menstruation period in reliable cases. Pregnant women were examined in lithotomy position, leakage of fluid was inspected by sterile speculum and only positive results were included. The diagnosis was confirmed by ultrasound examination to evaluate four quadrant amniotic fluid indexes.

Collection of biological material: It was done with the patient lying in the lithotomy position with good illumination. After confirming absence of vaginal bleeding, samples were obtained during speculum examination from all patients. A cotton tipped swab was placed first into the endo-cervical canal and then into the posterior fornix of vagina, each for $30 \mathrm{~s}$.

The swab was placed in a tube containing $1 \mathrm{ml}$ of saline solution and the tube was shaken for $1 \mathrm{~min}$ before the swab was disposed. The assay procedure began with centrifugation of the solution for $5 \mathrm{~min}$ to remove particulate matter.

Sample assays : Insulin like growth factor binding protein concentration was assessed by ELISA. Also, quantitative $\beta \mathrm{HCG}$ in cervicovaginal secretion by microplate immunoenzymometric assay was carried out. Morover, quantitative prolactin in cervicovaginal secretion by microplate immunoenzymometric assay was done on all patients.

Outcome : All speculum examinations were performed by the same obstetrician and all samples were studied in the same laboratory and by the same technique in order to eliminate inter-observer sampling difference.

\section{STATISTICAL ANALYSIS:}

Data entry and statistical analyses were performed using SPSS (statistical package of social sciences) version 21 (SPSS Inc., Chicago, IL, USA by baron and Kenny 1086). Categorical data were expressed in number and percentage. Continuous normally distributed data were expressed in mean and standard deviation. The quantitative data were examined by Kolmogrov Smirnov test for normality of data ${ }^{[16]}$.

Student T-test was used for comparing continuous normally distributed data. Comparing of categorical data was done using chi square test or fisher exact test used whenever appropriate.

Sensitivity, specificity, diagnostic efficiency, positive and negative predictive value were calculated and the overall diagnostic performance of the test was assessed by receiver-operating characteristics (ROC) curve analysis. Statistical significance was considered when probability $(\mathrm{P})$ value was less than or equal to 0.05 .

Receiver-operator characteristic (ROC): Curves were calculated the area under the curve (AUC) and 95\% confidence interval (CI) was calculated for each plot. In order to select cutoff value having the best combined sensitivity and specificity for each marker (Fig. 1)

Stepwise approach was performed. The first step is to determine the accuracy of each marker alone. Second step was examining the diagnostic accuracy of markers combinations in pairs and computing each pair as if it was a new marker.

Next step was to find the pair if markers whose optimal linear combination is best in the sense of having maximal AUC (or pAUC) amongst all pairs of markers.

The advantage of the stepwise approach is that each step requires computation for only two markers at a time, giving better chance of comparing between combinations.

Hence, pairing $\beta$-HCG with prolactin offered lower diagnostic ability than each of them alone, it seemed illogic to proceed with combining all the three markers. 


\section{RESULTS}

In our study, the included women $(\mathrm{n}=180)$ between 28 to 36 weeks gestation of single viable fetus have been selected in the study, $\mathrm{n} 1$ (case group) $=90$ with confirmed rupture membranes and n2 (control group) $=90$ with no suggestive history of ruptured membranes.

The initial characteristics of both groups of women were that they had the mean maternal age $25.5 \pm 2$. 2 years for the study group and $27.1 \pm 3.4$ years for the control group, the mean GA at delivery was $31.7 \pm 3.1$ and $32.9 \pm 2.9$ weeks for the study and the control group, respectively (Table 1).

There was significant difference of prolactin, ILGFBP-1 and $\beta$-HCG concentration between group I (PROM) compared to group II (control) (Table 2).

Receiver operating characteristic (ROC) curve analysis was applied to assess the diagnostic performance of Prolactin $(\mu \mathrm{IU} / \mathrm{ml})$, ILGFBP I $(\mu \mathrm{g} / \mathrm{l})$ and $\beta$ hCG $(\mathrm{mIU} / \mathrm{mL})$, the area under the curve was $0.756,0.911,0.785$ for the three markers respectively, which is significant.

It means that each marker alone could predict the presence of amniotic fluid in vaginal fluid with sensitivity $\%$, specificity $\%$, PPV\% and NPV \% as shown in the table below.

The best cut off values to diagnose PROM were chosen to be $11 \mu \mathrm{IU} / \mathrm{ml}$ for prolactin, $102 \mu \mathrm{g} / 1$ for ILGFBP-1 and $104 \mathrm{mIU} / \mathrm{mL}$ for $\beta$-HCG.

In this study, same statistic characters were investigated by pairing every couple of the markers of choice and judging each pair as a new marker (Table 3).

This table showed the cut off values of each marker alone and each pair of markers and compares between the diagnostic accuracy of the six markers separately.

Table 1: Background characteristics of both groups

\begin{tabular}{lccc}
\hline Variable & PROM & Control & $P$ \\
\hline Maternal age (years) mean \pm SD & $25.5 \pm 2.2$ & $27.1 \pm 3.4$ & 0.104 \\
GA (weeks) mean \pm SD & $31.7 \pm 3.1$ & $32.9 \pm 2.9$ & 0.082 \\
\hline
\end{tabular}

P values: $0.003,0.004,0.001$

Table 2: Comparison between different outcomes in both groups

\begin{tabular}{lccc}
\hline Variable & Group I (Case) & Group II (Control) & $P$ \\
\hline Prolactin $(\mu \mathrm{IU} / \mathrm{ml})$ & $9.2 \pm 5.4$ & $4.2 \pm 2.5$ & $<0.001^{*}$ \\
ILGFBP-1 $(\mu \mathrm{g} / \mathrm{l})$ & $243.2 \pm 110.8$ & $76.6 \pm 32.9$ & $<0.001^{*}$ \\
$\beta$-HCG $(\mathrm{mIU} / \mathrm{mL})$ & $199.7 \pm 102.1$ & $64.8 \pm 29.6$ & $<0.001^{*}$ \\
\hline
\end{tabular}

P values: 0.003, 0.004, 0.001

Table 3: Accuracy of different parameters

\begin{tabular}{lcccccc}
\hline $\begin{array}{l}\text { Diagnostic } \\
\text { Accuracy }\end{array}$ & Prolactin & ILGFBP-1 & $\beta$-HCG & $\begin{array}{c}\text { ILGFBP-1+ } \\
\beta \text {-HCG }\end{array}$ & $\begin{array}{c}\beta \text {-HCG+ } \\
\text { prolactin }\end{array}$ & $\begin{array}{c}\text { Prolactin+ } \\
\text { ILGFBP-1 }\end{array}$ \\
\hline Cutoff & $11 \mu \mathrm{IU} / \mathrm{ml}$ & $102 \mu \mathrm{g} / 1$ & $104 \mathrm{mIU} / \mathrm{mL}$ & & & \\
AUC & 0.756 & 0.911 & 0.785 & 0.846 & 0.899 & 0.825 \\
Sensitivity \% & 84.4 & 88.3 & 85.1 & 78.4 & 79.8 & 78.4 \\
Specificity \% & 73.2 & 93.5 & 83.9 & 100 & 100 & 100 \\
PPV \% & 73.8 & 100 & 96.2 & 100 & 100 & 100 \\
NPV \% & 79.5 & 69.4 & 72.8 & 64 & 63.6 & 68.7 \\
Accuracy & 75.4 & 81.5 & 79.9 & 89.7 & 77.2 & 84.2 \\
95\% CI & $0.491-0.823$ & $0.691-0.881$ & $0.851-0.971$ & $0.664-0.904$ & $0.583-0.966$ & $0.885-0.979$ \\
\hline
\end{tabular}




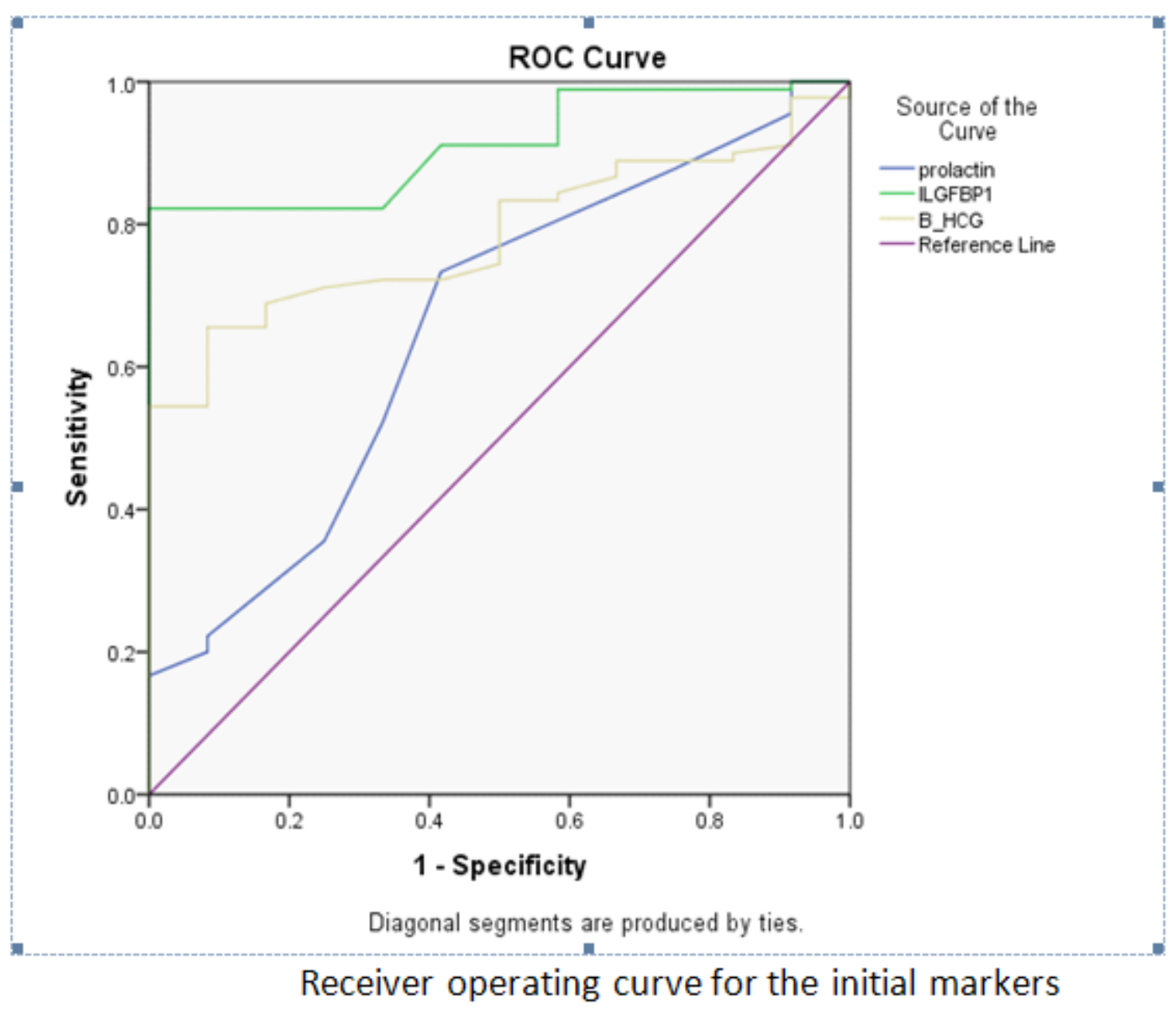

\section{DISCUSSION}

In the current study, there were no significant differences in demographic characteristics of both the cases and the control groups.

Diagnostic accuracy is generally assessed by the receiver operating characteristic (ROC) curve, which is defined as the plot of sensitivity against 1-Ispecificity over all possible cut off values. Different tests may be compared by the area under ROC curve (AUC), or the sensitivity at a fixed common specificity ${ }^{[17]}$.

As a single marker, IGFBP-1 was found in the current study to have sensitivity, specificity, PPV and NPV of IGFBP-1 with cut-off point $102 \mu \mathrm{g} / 1$ were $88.3,93.5,100$ and 69.4 , respectively, gives accuracy percentage $81.5 \%$.

Similar values are concluded $\mathrm{in}^{[7]}$. The study examined IGFBP-I concentration in samples obtained from the posterior fornix of 54 pregnant women with intact membranes, who had been attending for routine antenatal care between 24 and 40 weeks, against cervico-vaginal fluid of 35 pregnant women who had had spontaneous or artificial ruptured membranes. They interpreted the values greater than $100 \mu \mathrm{g} / \mathrm{l}$ as containing amniotic fluid. They reported sensitivity of $75 \%$ and a specificity of $97 \%$ in diagnosis of ROM. The positive predictive value was $95 \%$ for IGFBP-1 measurement.

Regarding the cut-off value ${ }^{[18]}$ used the actim PROM test (Medix Biochemical OY AB, Kauniainen, Finland), which interprets $25 \mu \mathrm{g} / 1$ as positive on 58 healthy pregnant women who were undergoing genetic amniocentesis at 15-20 weeks of gestation and concluded the sensitivity of the actim PROM test compared to that of other commercial tests. Interestingly, they concluded values close to the studies which used higher cut-off points. Their clinical evaluation was recorded as $85 \%$ just after the procedure. The rate of specificity was $98 \%$, PPV was $85 \%$ and NPV was $98 \%$. 
This discrepancy in the cut-off value could be elucidated by the huge difference in the main gestational age of the selected population.

On the contrary ${ }^{[19]}$, who used the same commercial kits that have been used by Sucak on 83 patients with clinically confirmed membranes state, the sensitivity, specificity, PPV and NPV are 81, 71, 89 and 58\%, respectively. Therefore, they concluded that detection of IGFBP-1 has no additional benefit for obstetricians to ascertain the diagnosis of ruptured fetal membranes in cases in which there is clinical doubts.

This conclusion might be attributed to the lower cut-off point that is employed by the actim PROM test, (Medix Biochemical OY AB, Kauniainen, Finland), which is almost one quarter of the cut-off value used by Rotanen et al. and the current study.

In the case of using prolactin solely, the results obtained by the current study revealed that the sensitivity, specificity, PPV and NPV of prolactin with cut-off point $11 \mu \mathrm{IU} / \mathrm{ml}$ are 84.4, 73.2, 73.8 and 79.5 respectively gives accuracy percentage $75.4 \%$.

That is compatible with ${ }^{[15]}$, who stated that at cutoff value of $9.50 \mu \mathrm{IU} / \mathrm{ml}$ the sensitivity, specificity, positive and negative predictive values and accuracy of vaginal fluid prolactin as a marker of ruptured membranes had been $87.03 \%, 75.0 \%, 75.80 \%, 86.53 \%$ and $83.33 \%$, respectively, after they examined a total of 114 pregnant women falling into two groups; PROM and control.

However ${ }^{[20]}$, in their study, which included 80 women who were divided into two groups (confirmed rupture membranes and intact membranes) mentioned that vaginal wash prolactin at cut-off value level of $659 \mathrm{ng} / \mathrm{l}$, which equals $14 \mu \mathrm{IU} / \mathrm{mL}$, could significantly predict the occurrence of PROM with diagnostic sensitivity, specificity, positive predictive value (PPV), and negative predictive value (NPV) of $70.0 \%, 95.0 \%, 93.3 \%, 76.0 \%$, respectively.

Elevating the cut-off point seems to enhance the specificity on the account of lowering the sensitivity

Although a relatively higher figure was concluded as cut-off value by ${ }^{[21]}$, who compared four biomarkers in diagnosing premature rupture of membranes in 80 women divided in two groups and set their cut-off value at $23.56 \mu \mathrm{IU} / \mathrm{ml}$ for prolactin. They concluded less beneficial results sensitivity, specificity, PPV, NPV and efficiency 78\%, 68\%, 70.9\%, 75.56\% and $73 \%$, respectively.
This difference in their results might be explained by the high percentage of their patients who had established true labour pain (three contractions in ten minutes) within the following few hours.

Regarding the last marker to be used in the current study ; $\beta-\mathrm{HCG}$, the sensitivity, specificity, PPV and NPV of $\beta$-HCG with cut-off point $104 \mathrm{mIU} / \mathrm{mL}$ are $85.1,83.9,96.2$, and 72.8 , respectively, gives accuracy percentage $79.9 \%$.

Less optimistic values were concluded by ${ }^{[8]}$ study, in which they tested qualitative human chorionic gonadotropin (hCG) on a total of 92 pregnant women and the patients with negative, positive and suspected pooling were classified into three groups, (hCG) concentration $100 \mathrm{mIU} / \mathrm{mL}$ was the chosen cut-off value for positive ruptured membranes. The sensitivity, specificity, positive predictive and negative predictive values were calculated as $71.2,100,100$, and $65.1 \%$, respectively.

This conclusion may be due to the small number of candidates in each of their three groups. ${ }^{[22]}$ concluded that a B-HCG cut-off value of $79.5 \mathrm{mIU} / \mathrm{mL}$ by a research on 123 women divided into confirmed ruptured, suspected ruptured and intact membranes. Sensitivity of $95 \%$ and specificity of $84 \%$ had been determined to be optimal. The difference in the cutoff point could be attributed to the patient selection of their cases, which included suspected cases beside the confirmed cases of rupture membranes.

In a study by ${ }^{[23]}$, analysis was performed on 177 pregnant women with confirmed membranes status, the major cut-off point studied was of 100 $\mathrm{mUI} / \mathrm{mL}$, and it provided a sensibility of $71.2 \%$, a specificity of $100 \%$, a PPV of $100 \%$, and an NPV of $65.1 \%$; even though there was a high specificity with this cut-off point, it showed lower sensibility.

On the contrary ${ }^{[24]}$, claimed that using commercial kits with cut-off value $20 \mathrm{mIU} / \mathrm{ml}$ had provided sensitivity $86.66 \%$, specificity $96.66 \%$, PPV $96.29 \%$ and NPV $=87.87 \%$. They proposed that diagnostic accuracy of test had been $91.66 \%$ when taking beta HCG concentration at $20 \mathrm{mIU} / \mathrm{ml}$. In this study, exclusion criteria, which were not mentioned, might have explained the difference in the results.

When multiple tests are involved, the performance of the diagnosis may be improved by combining several tests as a new composite diagnostic test since different tests may be sensitive to different aspects of case $^{[14]}$. 
Combining results of diagnostic tests will result in a trade-off in sensitivity and specificity of the combined test relative to the component tests. The trade-off in the expected number of additional true positive and false positive results (or true negative and false negative results) is used as the basis for deciding whether to use tests in combination when neither the combined nor a component test shows superior test performance based on their likelihood ratios ${ }^{[25]}$.

In this study, the sensitivity, specificity, PPV, and NPV of the combination of IGFBP-1 and $\beta-\mathrm{HCG}$ are $78.4,100,100$ and 64 respectively gives accur acy percentage 89.7. This combination elevated the accuracy to 89.7 compared to 81.5 for IGFBP alone and 79.9 for $\beta$-HCG alone, and elevated specificity to 100 and PPV to $100 \%$.

This combination of IGFBP- 1 and $\beta-\mathrm{HCG}$ showed the best accuracy and diagnostic value compared to the other combinations.

The sensitivity, specificity, PPV and NPV of the combination of IGFBP-1 and prolactin are 78.4, 100, 100 and 68.7, respectively, gives accuracy percentage 84.2 . This combination mildly elevated the accuracy to only 84.2 against 81.5 for IGFBP-1 and 75.4 for prolactin, and elevated specificity to 100 and PPV to $100 \%$.

The diagnostic value of combining IGFBP-1 and prolactin is better than each test of the three markers alone, but not far better than the accuracy of the IGFBP-1 alone.

The sensitivity, specificity, PPV, and NPV of the combination of $\beta-\mathrm{HCG}$ and prolactin are $79.8,100,100$ and 63.6, respectively, gives accuracy percentage 77.2. This combination decreased the accuracy from 75.4 for prolactin alone and 79.9 for $\beta$-HCG alone to 77.2 for the combination but elevated specificity to 100 and PPV to $100 \%$.

Adding prolactin to $\beta-\mathrm{HCG}$; although being the cheapest combination, proposed lower accuracy than each of the other two markers alone.

Since combining markers could elevate the specificity on the account of sensitivity, while, on the contrary, decreasing the cut-off value enhances the sensitivity on the account of specificity, selecting lower cut-off points of the combined tests might offer benefits in both of the sensitivity and specificity.

Being cheap and available, and owing fair accuracy, $\beta$-HCG alone can be employed as a risk factor along with history of vaginal infection and query clinical examination.
Those patients with all the risk factors positive are good candidates for performing combined test to detect amniotic fluid traces in the vagina. Patients who demonstrate positive combined test need level up investigations. This level up can include ultrasound evaluation, infection screening, evaluation of and accelerating (if feasible) fetal maturity, preparing for delivery -as PROM at any gestational age may be associated with brief latency from membrane rupture to delivery- and checking the accessibility of neonatal intensive care unit.

\section{CONCLUSION}

In query membrane state, combining IGFBP-1 and $\beta-H C G$ is the best diagnostic combination to detect amniotic fluid presence in vaginal fluid. Combined IGFBP-1 and prolactin is a little superior to each of the three markers (IGFBP-1, prolactin and $\beta-\mathrm{HCG}$ ) alone, but the offered improvement is very little compared to IGFBP-1 alone. Adding prolactin to $\beta$-HCG decreased accuracy than each of the other two markers alone.

\section{CONFLICT OF INTEREST}

There are no conflicts of interests.

\section{REFERENCES}

1. Parfieniuk E, Zbucka-Kretowska M, Ciborowski M, Kretowski A, and Barbas C (2018): Untargeted metabolomics: an overview of its usefulness and future potential in prenatal diagnosis. Expert Review of Proteomics; 15(10):809-16.

2. Cunningham FG, Leveno KJ, Bloom SL, Dashe JS, Hoffman BL, Casey BM and Spong CY (2018): Implantation and Placental Development, In: William's obstetrics $25^{\text {th }}$ ed., McGraw Hill ed.; (5):68-82.

3. Simhan HN, Iams JD, Romero R, Gabbe SG, Simpson JL, Landon MB, Galan HL, Jauniaux ERM, Driscoll DA, Berghella V and Grobman WA (2017): Preterm Labor and Birth, In: Obstetrics Normal and Problem Pregnancies $7^{\text {th }}$ ed., Elsevier, Inc.; (2):615-40.

4. Mercer BM, Gabbe SG, Simpson JL, Landon MB, Galan HL, Jauniaux ERM, Driscoll DA, Berghella V and Grobman WA (2017): Premature Rupture of the Membranes, In: Obstetrics Normal and Problem Pregnancies $7^{\text {th }}$ ed., Elsevier, Inc.; (2):640-65.

5. Abdelazim I, Al-Sherbeeny M, Ibrahim M, Fahmy A, Rabei N and Khalifa A (2016): Insulin-like Growth Factor Binding Protein-1/ 
alpha-fetoprotein versus Placental alpha microglobulin-1 for Diagnosis of Premature Fetal Membranes Rupture, Acta Medica International, Elsvier; 3(1): 69-74.

6. Smith RP and Netter FH (2018): Chorioamnionitis, In: Netter's Obstetrics and Gynecology, $3^{\text {rd }}$ ed. by Elsevier, Inc Netter; 464-9.

7. Rutanen E-M, Pekonen $\mathrm{F}$ and Käirkkäinen $\mathrm{T}$ (1993): Measurement of insulin-like growth factor binding protein-I in cervical/vaginal secretions: comparison with the ROM-check Membrane Immunoassay in the diagnosis of ruptured fetal membranes, Clinica Chimica Acta, Elsevier; 73-81.

8. Temel O, Çöğendez E, Selçuk S, Asoğlu M, Kaya E (2013): $\beta$-human chorionic gonadotropin assay in vaginal washing fluid for the accurate diagnosis of premature rupture of membranes during late pregnancy, J Turkish-German Gynecol Assoc; 14:201-4.

9. Korevaar TI, Steegers EA, de Rijke YB, Schalekamp-Timmermans S, Visser WE, Hofman A, Jaddoe VW, Tiemeier H, Visser TJ, Medici M and Peeters RP (2015): Reference ranges and determinants of total hCG levels during pregnancy: the Generation R Study. Eur J Epidemiol; 30(9):1057-66.

10. Huang $X$, Zhang $H$, Guo $X$, Zhu Z, Cai H and Kong X (2018): Insulin-like growth factor 2 mRNA-binding protein 1 (IGF2BP1) in cancer, In: Journal of Hematology and Oncology; 11:88.

11. Levine S and Muneyyirci-Delale O (2018): StressInduced Hyperprolactinemia: Pathophysiology and Clinical Approach, In: Obstetrics and Gynecology International; vol 2018, Article ID 9253083, 6 pages.

12. Su JQ and Liu JS (1993): Linear combination of multiple diagnostic markers. Journal of the American Statistical Association; 88: 1350-5.

13. Pepe MS, Cai TX and Longton G (2006): Combining predictors for classification using the area under the receiver operating characteristic curve Biometrics; 62:221-9.

14. Naaktgeboren CA, Bertens LCM, Smeden M, de Groot JAH, Moons KGM, Reitsma JB (2013): Value of composite reference standards in diagnostic research BMJ; 347:f5605.
15. Kariman N, Hedayati $M$ and Majd A (2012): The diagnostic power of cervico-vaginal fluid prolactin in the diagnosis of premature rupture of membranes, Iran Red Crescent Med J; 14(9):541-8.

16. Dean F (2006): Statistical methods in scientific researches. Europian Journal of Scientific Research; 14(3).

17. Metz CE (2006): Receiver Operating Characteristic Analysis: A Tool for the Quantitative Evaluation of Observer Performance and Imaging Systems. Journal of the American College of Radiology; 3(6): 413-22.

18. Sucak A, Moroy $\mathrm{P}$, Cakmak $\mathrm{P}$, Mungan $\mathrm{T}$, Mollamahmuto L, Danifiman N (2005): InsulinLike Growth Factor Binding Protein-I; a Rapid Detection of Amniotic Fluid Leakage after Amniocentesis Turk J Med Sci.; 35:157-61.

19. Jeurgen-Borst AJ, Bekkers RL, Sporken JM, van den Berg PP (2002): Use of insulin like growth factor binding-1 in the diagnosis of ruptured fetal membranes. Eur J Obstet Gynecol Reprod Biol.; 10: 102 (1): 11-4

20. Abd El Fattah EA, El Zaher Karkour TA, Nasrat $\mathrm{R}$ and Khalil MM (2016): Role of vaginal wash prolactin, lactate, and ceruloplasmin in diagnosis of premature rupture of membranes. The New Indian Journal of OBGYN; 3(1): 9- 19.

21. Tigga MP and Malik S (2015): Comparative analysis of four biomarkers in diagnosing premature rupture of membranes and their correlation with onset of labour Int $\mathrm{J}$ Reprod Contracept Obstet Gynecol.; 4(4):1070-5.

22. Bahasadri S, Kashanian M and Khalili S (2013): Evaluation of vaginal fluid b-human chorionic gonadotrophin for the diagnosis of preterm premature rupture of membranes, J. Obstet. Gynaecol. Res.; 39(4):777-82.

23. Dartibale CB, Uchimura NS, Nery L, Schumeish AP, Uchimura LYT, Santana RG and Uchimur $T$ (2017): Qualitative etermination of Human Chorionic Gonadotropin in Vaginal Washings for the Early Diagnosis of Premature Rupture of Fetal Membranes, Rev Bras Ginecol Obstet.; 39:317-21.

24. Azam A, Husnain HM and Marryum I (2018): Premature rupture of membranes; Diagnostic 
accuracy of B-HCG test in vaginal washings taking amniotic fluid pooling as gold standard of diagnosing pron. Professional Med J; 25(2): 168-72.
25. Macaskill P, Walter S, Irwig L and Franco E (2002): Assessing the gain in diagnostic performance when combining two diagnostic tests, Statist. Med.; 21:2527-46. 